\title{
Ionic current magnetic fields in 3D finite-length nanopores and nanoslits
}

\author{
Seyed Ali Tabatabaei ${ }^{1}$, Abraham Mansouri $^{2}$, Ali Tarokh ${ }^{3}$, Seyed Farshid Chini ${ }^{1, \mathrm{a}}{ }_{(\mathbb{C}}$ \\ ${ }^{1}$ School of Mechanical Engineering, College of Engineering, University of Tehran, Tehran, Iran \\ 2 Department of Mechanical Engineering, HCT, DBM, Dubai, UAE \\ ${ }^{3}$ Department of Mechanical Engineering, Lakehead University, Thunder Bay, ON, Canada
}

Received: 15 November 2021 / Accepted: 23 February 2022

(C) The Author(s), under exclusive licence to Società Italiana di Fisica and Springer-Verlag GmbH Germany, part of Springer Nature 2022

\begin{abstract}
Deoxyribonucleic acid (DNA) encodes all genetic information, and in genetic disorders, DNA sequencing is used as an effective diagnosis. Nanopore/slit is one of the recent and successful tools for DNA sequencing. Passage of DNA along the pores creates non-uniform ionic currents which creates non-uniform electric and magnetic fields, accordingly. Sensing the electric field is usually used for sequencing application. We suggest to use the magnetic field induced by pressure-driven ionic currents as a secondary signal. We systematically compared the induced magnetic field of nanopores and nanoslits with equal cross-sectional area. The 3D magnetic field is numerically obtained by solving the Poisson-Nernst-Planck, Ampere, and Navier-Stokes equations. As expected, the maximum value of the maximum magnetic flux occurs near the wall and inside the channel, and increasing the pressure gradient along the pore/slit increases the flowrate and magnetic field, consequently. At a given pressure difference across the pore/slit, nanopores are better than nanoslits in sensing the magnetic flux. For example, by applying $2 \mathrm{MPa}$ across the pore/slit, the maximum magnetic flux density for nanopore, nanoslit $A R=1$ and nanoslit $A R=5$ are $1.10 \mathrm{pT}, 1.08 \mathrm{pT}$ and $0.45 \mathrm{pT}$, accordingly. Also, at a given flowrate across the pore/slit, nanoslits are the better choice. It should be noted the external magnetic fields as small as pico-Tesla are detectable and measurable in voltage/pressure driven electrokinetic flow slits.
\end{abstract}

\section{Introduction}

By deoxyribonucleic acid (DNA) sequencing, our understanding of disease, heredity, and individuality will improve. Genomes' sequence has been crucial in identifying genetic risk factors associated with complex human diseases and continues to play an evolving role in the treatment and personalized medicine [1]. DNA sequencing in forensic biology and biotechnology is an active field of research and motivated researchers to develop effective techniques for DNA tracking and stimulated critical technological innovations [2]. Such single-molecule studies yield a wealth of information on the dynamics and structure of oligonucleotides.

One of the methods of DNA sequencing is the use of nanopores. Translocation of biological matters (e.g., DNA, RNA, nucleic acids, or proteins) along a nanopore enables us to analyze the biological properties with high throughput and resolution [3, 4]. Utilizing nanopores is a label-free, amplification-free, single-molecule approach that can be scaled for high-throughput DNA analysis. Moreover, nanopore DNA sequencing requires minimal sample volumes and does not need the use of purified fluorescent reagents nor amplified genomic DNA, i.e., eliminates enzymes, cloning, and amplification steps [5]. So, it has the potential for integration into small and portable devices for low-cost DNA analysis [6, 7]. In addition, nanopores with suitable sizes and properties can be utilized to detect and count various viruses such as influenza and COVID-19 [8-10]. Many researchers have used different characteristics of nanopore systems for sequencing application, e.g., the current-voltage (I-V), current density, electric field density, electrostatic potential distributions, and pressure [11-17].

Recently, with the development of solid-state nanopore/slit fabrication, ultrathin nanopores have been produced and explored [18-22]. These ultrathin solid-state nanopores provide improved sensitivity and spatial resolution in sensing applications. They can also work over a broader range of temperature, $p \mathrm{H}$, and chemical environments and potentially be integrated into practical devices or platforms. Solid-state nanopores/slits can also be customized in mechanical, chemistry and thermal properties, as well as size, and geometry (e.g., nanoslit [23-25], nanosqaure, and nanotringle, [26, 27]); while their biological counterparts cannot [28-30]. The other advantage of using solid-state nanopores/slits is the longer lifetime. Also, solid-state nanopores/slits allow additional device complexity and incorporation of additional tools [29-31].

In nanopores/nanoslits, once a small voltage bias is enforced across the pore, the nanopore acts as a membrane separating two aqueous electrolyte chambers. Therefore, it works as a biosensor and provides a particular passage through which the ionic solutions on the cis (entry) and trans (exit) sides of the membrane are connected. A continuous voltage bias (trans side positive) produces an ionic current through the pore and drives single-stranded DNA (ssDNA) or single-stranded RNA (ssRNA) from the

\footnotetext{
a e-mail: chini@ut.ac.ir (corresponding author)
} 
cis chamber through the pore to the trans chamber. The resulting ionic current through the pore can be measured with standard electrophysiological techniques [32-35]. The passage of each DNA molecule can be detected as a temporary decrease of ionic current (current blockade), and blockade duration is proportional to the DNA length. By measuring the duration and magnitude of the blockade, the length of ssDNA can be determined [36-39]. As a result, these systems are potentially able to distinguish short-lived DNA and RNA molecules, which have a helical diameter of 2-3 nm and a length of about $136 \mathrm{~nm}$, without losing the signal-to-noise and capture rate ratios [40, 41].

In the measurement of ionic current blockades as ssDNA or ssRNA passes through a nanopore/nanoslit, the super-fast speed of translocation through the nanopore, small current value, and short current blockade time make the measurements quite challenging. Thus, a crucial challenge to nanopore DNA sequencing is to slow down and control DNA/RNA translocation velocity and reduce the variations in translocation kinetics due to the pore-surface interactions [42, 43]. The following parameters may be utilized to slow down the translocation velocity: pore size, length and shape, electric field, viscosity of the solution, ionic concentration, orientation and conformation of the molecule, and interaction between DNA/RNA and the pore wall [30, 36, 38]. Also, creating an opposing force can be helpful to slow down the translocation velocity, e.g., pressure gradients (pressure-voltage trap) [44, 45]. Another major challenge in nanopore/nanoslit RNA/DNA sequencing is the sensitivity of the approach, which can be resolved by new sensing modalities, reduction of noise, new device architectures, or improvements in fabrication technology [12].

We believe the challenges about the sensitivity of RNA/DNA sequencing approach may be addressed by using magnetic fields as the secondary signal (the primary signal is electric field). Sensing external magnetic field generated by the pressure-driven ionic currents may be used as a non-invasive technique to complement/enhance sensitivity, improve the rate of sequencing, and make DNA and RNA nanopore/nanoslit sequencing more accurate. This research is a continuation of our previous studies [46, 47]. In this work, using a multi-physics FEM model, for the first time, the magnetic field created by the ionic current through 3D nanoslits/nanopores is studied. The 3D model enables us to compare the nanoslit and nanopore geometries quantitatively. The pressure-driven electrokinetic flow in nanoslits/nanopores is simulated by modified Navier-Stokes and Poisson-Nernst-Planck equations. We clarify the understanding of ionic current and induced internal and external magnetic fields in charged finite length 3D nanopores/nanoslits.

\section{Problem statement}

The steady-state flow of an electrolyte solution is considered in 3D nanoslit/nanopore with dimensions shown in Fig. 1a, b. The cross-sectional area of nanopore and nanoslit are equal, and nanoslit aspect ratio $(A R)$ is defined as the ratio of slit width $(w)$ to height $(h)$, see Fig. 1. Note the width of a nanoslit with $A R=1.27$ is equal to the diameter of the nanopore.

The electrolyte solution is bounded by nanopore/nanoslit walls made of dielectric materials. Due to the interaction between the wall material and electrolyte solution, a surface potential (zeta potential) is formed near the walls. The finite length geometry of the nanopore/nanoslit allows the creation of suitable ion composition and flux at the nanopore/nanoslit entrance and exit. The reservoir walls are far from the nanopore/nanoslit entrance or exit as done in [48] to minimize the effect of reservoir walls on the ion composition inside the nanopore/nanoslit. It should be mentioned that such assembly was adopted by Mansouri et al. for even unsteady problems [49-51].

To focus on the formation of magnetic fields and comparison with analytical models, the wall thickness is considered zero, impermeable to mass and electric field but permeable to temperature and magnetic field. The nanoslit/nanopore is surrounded by air (CDEF area in Fig. 1c), which is then analyzed to capture external magnetic fields.

\section{Governing equation}

The electro-hydrodynamic transport inside nanoslits/nanopore was modeled by solving the governing equations of Poisson-NernstPlanck, Ampere, and Navier-Stokes (i.e., P-N-P-A-N-S) simultaneously. The general form of the steady-state Navier-Stokes equation with the electrical body force is as follows:

$$
\rho(\mathbf{u} \cdot \nabla \mathbf{u})=-\nabla p+\mu \nabla^{2} \mathbf{u}-\rho_{f} \nabla \psi
$$

where $\rho$ is the fluid density $\left(\mathrm{kg} / \mathrm{m}^{3}\right), \mathbf{u}$ is the $3 \mathrm{D}$ velocity vector $(\mathrm{m} / \mathrm{s}), p$ is hydrostatic pressure $(\mathrm{Pa}), \mu$ is the viscosity of the fluid (Pa.s), $\rho_{f}$ is the free electrical charge density $\left(\mathrm{C} / \mathrm{m}^{3}\right)$, and $\psi$ is the electrical potential $(\mathrm{V})$. The $\rho_{f}$ is calculated as:

$$
\rho_{f}=\sum e v_{i} n_{i}
$$

where $e$ is the elementary charge $\left(1.602 \times 10^{-19} \mathrm{C}\right)$, and $v_{i}$ and $n_{i}$ are the valence and number concentration $\left(\frac{1}{m^{3}}\right)$ of the $i^{\text {th }}$ electrolytic species. It is assumed there is no external magnetic field source, also the impact of magnetic fields (induced by small ionic currents) are not significant on fluid flows inside the nanoslits/nanopores [52]. 


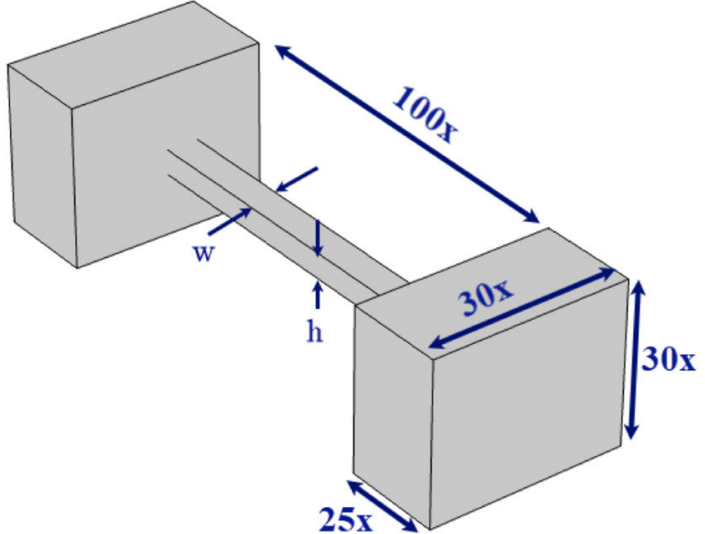

$A R=w / h$

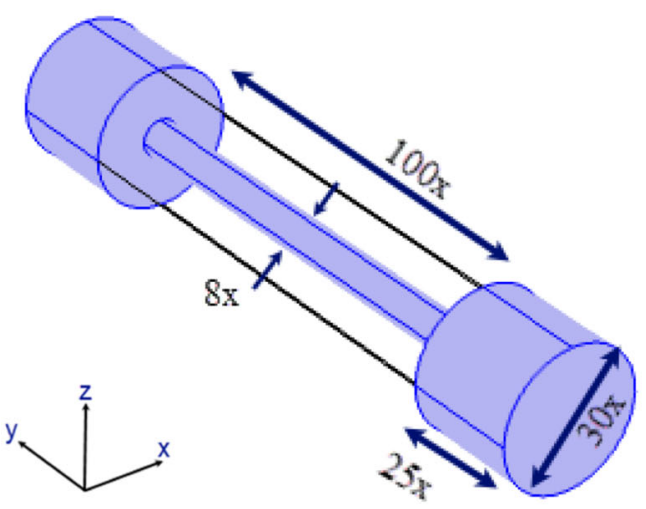

(b)

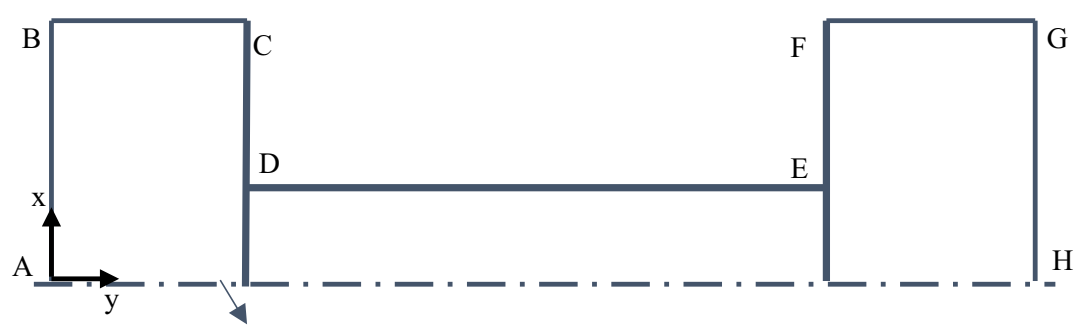

Symmetry line for nanoslit, and axisymmetric line for nanopore

(c)

Fig. 1 Schematic of 3D finite length a nanoslit and $\mathbf{b}$ nanopore connecting the two finite reservoirs, and $\mathbf{c}$ the side-view schematic of the nanoslit/nanopore geometry

The Poisson-Nernst-Planck (PNP) equations were used to estimate charge distribution, electric potential, and ion transport. The electric potential is associated with free charge density by the Poisson equation [50]:

$$
\nabla^{2} \psi=-\frac{\rho_{f}}{\epsilon}
$$

where $\epsilon$ is the dielectric permittivity of the fluid $(\mathrm{F} / \mathrm{m})$. In the electrolyte solution, the total flux for ion transport $\boldsymbol{J}_{i}\left(\frac{1}{\mathrm{~m}^{2} s}\right)$ is calculated by the Nernst-Planck equation. As total flux of ions in the solution, i.e., $\boldsymbol{J}_{i}$, is characterized by sum of convective, diffusive, and migratory fluxes:

$$
J_{i}=n_{i} u-D_{i} \nabla n_{i}-\frac{e v_{i} n_{i} D_{i}}{k_{B} T} \nabla \psi
$$

where $D_{i}$ is the diffusivity of the $i$ th electrolytic species $\left(\frac{m^{2}}{s}\right), k_{B}$ is Boltzmann's constant $\left(1.38 \times 10^{-23} \mathrm{~J} / \mathrm{K}\right)$ and $T$ is temperature (K). Moreover, as the electrolyte solution is dilute, all fluid properties are considered to be uniform and constant throughout the liquid domain. In the simulations, no time-dependent terms were kept in the governing transport equations.

The free flow of electrons or ionic species can create magnetic fields inside and around the nanoslit/nanopore. Here, the creation of magnetic flux by the ionic current density $\left(\boldsymbol{J}=\sum \boldsymbol{J}_{\boldsymbol{i}}\right)$ will be examined. Conservation of current, or the equation of current continuity, is given by:

$$
\nabla \cdot J=0
$$

According to Ampere's circuital law, the magnetic field strength is related to the current density:

$$
\nabla \times \mathcal{H}=\mathfrak{J}
$$

where $\mathcal{H}$ is the magnetic field strength $\left(\frac{A}{m}\right)$ and $\mathfrak{J}$ is current density $\left(\frac{A}{m^{2}}\right)$. The magnetic flux density is related to the magnetic field strength as:

$$
\mathfrak{B}=\mu_{0} \mu_{r} \mathcal{H}
$$


where $\mathfrak{B}$ is the magnetic field density $(\mathrm{T}), \mu_{0}$ is the vacuum permeability $\left(4 \pi \times 10^{-7} \mathrm{~N} / \mathrm{A}^{2}\right)$, and $\mu_{r}$ is the relative permeability, e.g., for air $\mu_{r} \sim 1$. There are two limiting steady-state cases for pressure-driven flows inside charged nanoslits/nanopores. For the problem stated in this paper, as the external force disturbs the double layer, two interrelated electrokinetic phenomena may occur. By forcing the electrolyte solution through the pore/slit, the ionic charges in the movable part of the electric double layer near the walls are convected with the liquid flow; such a current constitutes the streaming current (SC). In the absence of an external electric connection between the inlet and outlet, the accumulation of charge creates an electric field. As the electric field builds up, a current flow in the opposite direction starts to appear. At steady-state conditions, the net current is zero, and as the external circuit is open, the inequality of net charges in the locale of input and output of nanoslit/nanopore creates a streaming potential (SP). The streaming current creation of net charge density and velocity field can be represented as:

$$
\boldsymbol{J}=\left(n_{p}-n_{n}\right) \boldsymbol{u}
$$

where $n_{p}=\sum e v_{i} n_{i}$ is summed only over the positively charged species, and $n_{n}$ is the same but summed over the negative species. As the streaming potential generates a conduction current of a comparable magnitude contrary to streaming current and assuming diffusion current is negligible, the total flux of ions in the solution is given by:

$$
\boldsymbol{J}=\left(n_{p}-n_{n}\right) \boldsymbol{u}+\frac{\left(n_{p}-n_{n}\right) D}{K_{B} T} \nabla \psi,
$$

where $D$ is the diffusivity of electrolyte. In the streaming potential mode, the two terms in the right-hand side of Eq. 9 have opposite signs resulting in a null net current.

\subsection{Non-dimensional equation}

To generalize the solution, all governing equations are converted to dimensionless form. The characteristic length is chosen to be the Debye length, for symmetric binary electrolytes [50]:

$$
\kappa^{-1}=\left(\frac{\epsilon k_{B} T}{2 n_{\infty} e^{2} v^{2}}\right)^{1 / 2}
$$

where $\kappa^{-1}$ is Debye length $(\mathrm{m}), v$ is valence number, and the value of all the parameters are listed in Table 2 . The diffusivities of negative and positive ions in the electrolyte solution are assumed to be equal (henceforth, $D_{i}=D$ ). While it is quite straightforward to include different diffusivities of ions in the numerical model.

The non-dimensional and scaled parameters are listed in Table 2.

The dimensionless form of the Navier-Stokes and Poisson equations can be obtained as follow:

$$
-\bar{\nabla} \bar{p}+\bar{\mu} \bar{\nabla}^{2} \overline{\boldsymbol{u}}-0.5\left(\bar{n}_{p}-\bar{n}_{n}\right) \bar{\nabla} \psi_{d}=0
$$

and

$$
\bar{\nabla}^{2} \psi_{d}=0.5\left(\bar{n}_{p}-\bar{n}_{n}\right)
$$

The non-dimensional Nernst-Plank equations for each ionic species for the positive and negative ions, respectively, are:

$$
\begin{aligned}
& -\bar{\nabla} \cdot\left(\bar{n}_{p} \overline{\boldsymbol{u}}-\bar{\nabla} \bar{n}_{p}-\bar{n}_{p} \bar{\nabla} \psi_{d}\right)=0 \\
& -\bar{\nabla} \cdot\left(\bar{n}_{n} \overline{\boldsymbol{u}}-\bar{\nabla} \bar{n}_{n}-\bar{n}_{n} \bar{\nabla} \psi_{d}\right)=0
\end{aligned}
$$

The non-dimensional form of Ampere's Law is given as:

$$
\bar{\nabla} \times \overline{\mathcal{H}}=\overline{\mathfrak{J}}
$$

\section{Numerical methods}

\subsection{Methods}

In the present work, a fully coupled finite element solver, integrated into COMSOL Multiphysics (V. 5.5), is used to solve the governing nonlinear PDEs for the steady-state condition. We have used Laminar flow, transport of diluted species, electrostatics, and magnetic field physics in COMSOL Multiphysics software. This software is based on the finite element method, which has proved its effectiveness over other numerical methods in the context of modeling of electromagnetic phenomena [53]. The results represent a coupled solution of the (PNPANS) equations in a 3D computational domain. Due to the physics of the problem, near the nanoslit/nanopore walls, where the electric field and flow field are pronounced, a refined computational mesh was used to ensure accurate modeling of field gradients. Near the nanoslit/nanopore centerline, velocities were comparatively large and highly 
Table 1 Physical parameters used to normalize the different values related to the problem

\begin{tabular}{ll}
\hline$e$ & $1.6021 \mathrm{e}-19[\mathrm{C}]$ \\
$\rho$ & $10^{3}\left[\mathrm{~kg} \mathrm{~m}^{-3}\right]$ \\
$n_{\infty}$ & $6.022 \mathrm{e} 24\left[\mathrm{~m}^{-3}\right]$ \\
$\epsilon$ & $78.5 \times 8.854 \mathrm{e}-12\left[\mathrm{CV}^{-1} \mathrm{~m}^{-1}\right]$ \\
$v$ & 1 \\
$T$ & $298[\mathrm{~K}]$ \\
$k_{B}$ & $1.38 \mathrm{e}-23\left[\mathrm{~J} \mathrm{~K}^{-1}\right]$ \\
$D$ & $10^{-9}\left[\mathrm{~m}^{2} \mathrm{~s}^{-1}\right]$ \\
Surface potential $(\xi)$ & $-50\left[\mathrm{mV}^{-1}\right.$ \\
\hline
\end{tabular}

Table 2 Non-dimensional and scaled parameters

\begin{tabular}{lll}
\hline X coordinate $(\bar{x}): \kappa x$ & Y coordinate $(\bar{y}): \kappa y$ & Z coordinate $(\bar{z}): \kappa z$ \\
\hline Velocity $(\overline{\boldsymbol{u}}): \frac{1}{\kappa D} \boldsymbol{u}$ & Density $(\bar{\rho}): \frac{v^{2} e^{2} D^{2}}{\epsilon k_{B}^{2} T^{2}} \rho$ & Pressure $(\bar{p}): \frac{v^{2} e^{2}}{\epsilon k_{B}^{2} T^{2} \kappa^{2}} p$ \\
Viscosity $(\bar{\mu}): \frac{v^{2} e^{2} D}{\epsilon k_{B}^{2} T^{2}} \mu$ & Electrical potential $(\bar{\psi}): \frac{v e}{k_{B} T} \psi$ & $\begin{array}{c}\text { Free charge density } \\
\left(\overline{\rho_{f}}\right): \frac{1}{v_{\infty} n_{\infty}} \rho_{f}\end{array}$ \\
$\begin{array}{c}\text { Ion concentration } \\
\left(\bar{n}_{p}, \bar{n}_{n}\right): \frac{n_{p}}{n_{\infty}} \cdot \frac{n_{n}}{n_{\infty}}\end{array}$ & Current $(\bar{I}): \frac{\kappa}{v e n_{\infty} D} I$ & Current density $(\overline{\boldsymbol{J}}): \frac{1}{v_{\infty} D \kappa} \boldsymbol{J}$ \\
$\begin{array}{l}\text { Magnetic field density } \\
(\overline{\mathfrak{B}}): \frac{v e D}{k_{B} T} \mathfrak{B}\end{array}$ & Magnetic field strength & \\
& $(\overline{\mathcal{H}}): \frac{1}{v e n_{\infty} D} \mathcal{H}$ & \\
&
\end{tabular}

dependent on the accuracy of the electric body force term, so a sufficiently refined mesh is needed to capture the elusive changes in the electric and magnetic fields. Due to the physics and geometry of the problem, an unstructured mesh was used, the grid was fine inside the pore/slit, and coarse away from the wall and outside the wall. The nanopore requires finer mesh due to its cylindrical shape, whereas the nanoslit geometry is more structured, i.e., 258,615 and 334,652 tetrahedral elements for nanoslit and nanopore, accordingly. Grid independency was checked so that the errors of flowrate and magnetic flux density become less than $1 \%$. The runtime of solving the nanopore problem with 334,652 elements is $7908 \mathrm{~s}$.

\subsection{Boundary conditions}

Figure 1c shows a 2D side view schematic of the nanoslit/nanopore geometry, in which the fluid flow is from left to right. The inlet flow boundary condition (A-B) is set to constant pressure, and the outlet pressure $(\mathrm{G}-\mathrm{H})$ is set to 0 , to create a continuous pressure-driven flowrate. Depending on the streaming current state, the inflow is also an electrically neutral electrolytic solute with a specified ion concentration $\left(n_{\infty}\right)$ at zero electrical potential. The boundary conditions for walls C-D, D-E, and E-F in Fig. 1c are non-slip and impermeable to both solvent and solute. Reservoir boundaries B-C and F-G are impermeable to solvent and solute, but they are assumed to be semi-infinite in simulation with complete slip conditions.

Due to the difficulty of modeling the electrode's electrochemistry and their polarization in streaming current phenomena, the outflow boundary $(\mathrm{G}-\mathrm{H})$ was set to zero electrical potential with specified ion concentration to satisfy neutral electrolyte conditions. The $-50 \mathrm{mV}$ surface potential was specified on the pore/slit walls, i.e., D-E in 2D view in Fig. 1, and zero on the other walls.

The dimensions for pore and slit are shown in Fig. 1a, b, and the calculations were performed using scaled nanoslit/nanopore dimensions. The physical parameters are shown in Table 1, and with such values, the Debye length becomes $3.03 \mathrm{~nm}$, and the cross-sectional area becomes $462 \mathrm{~nm}^{2}$. Although the pore size in this study may not sense the DNA/RNA blockage, the model is applicable to any geometry. In fact, this study is a first step to give the readers an insight into the possibility of the suggested methodology.

\subsection{Validation of numerical method}

The validity of numerical simulation is assessed using an analytical solution for ionic current in a straight and symmetric nanoslit of infinite length with specified surface potentials on the walls [15, 54]. Since the problem has not yet been investigated experimentally or in 3D, the 2D geometry of an infinite straight nanoslit is reconstructed, and the scaled velocity in the middle of nanoslit and both internal and external magnetic flux densities across the nanoslit width were compared with the analytical model. As shown in the "Appendix", for an infinite nanoslit in streaming current mode, the analytical and numerical velocity field and internal and external magnetic flux densities are in agreement (Table 2). 
Scaled electric potential

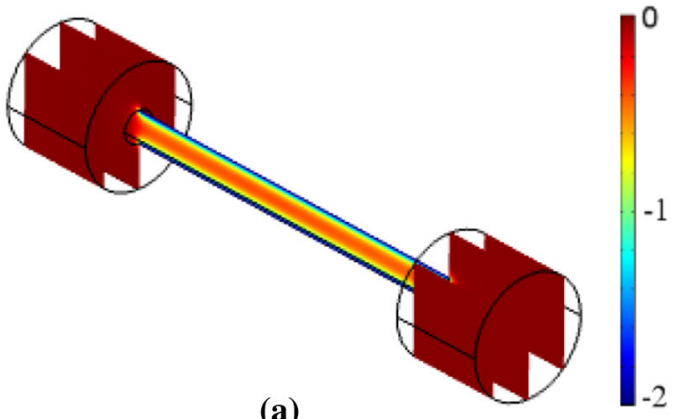

(a)

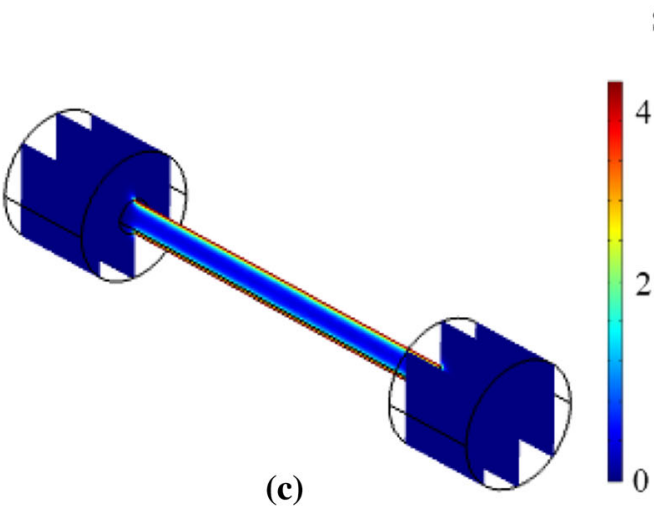

(c)

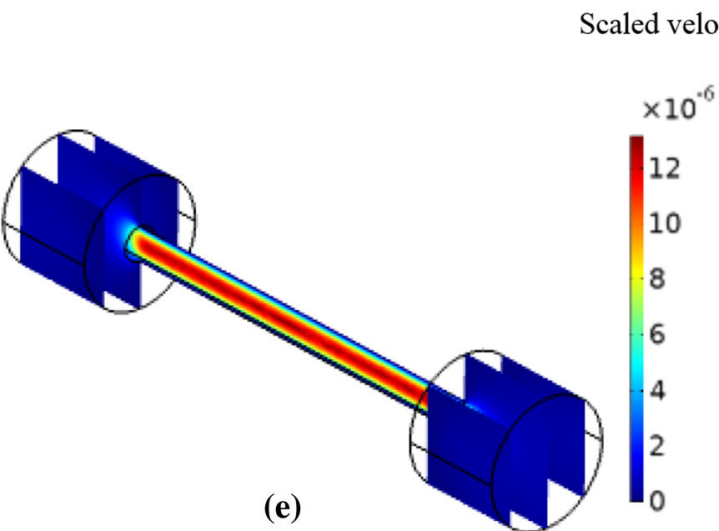

4

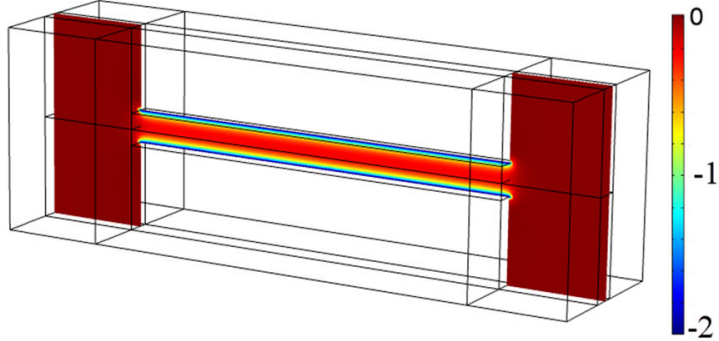

(b)

Scaled (np-nn)

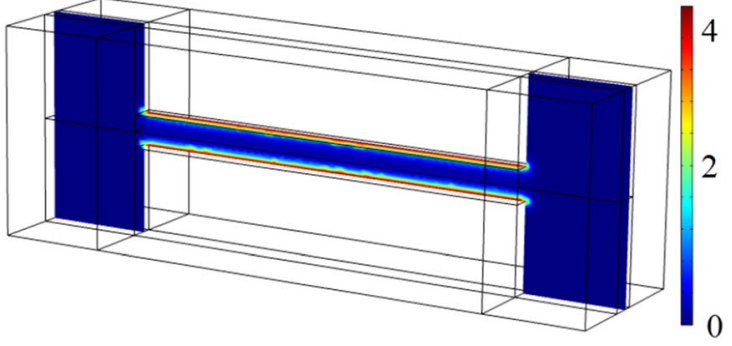

(d)

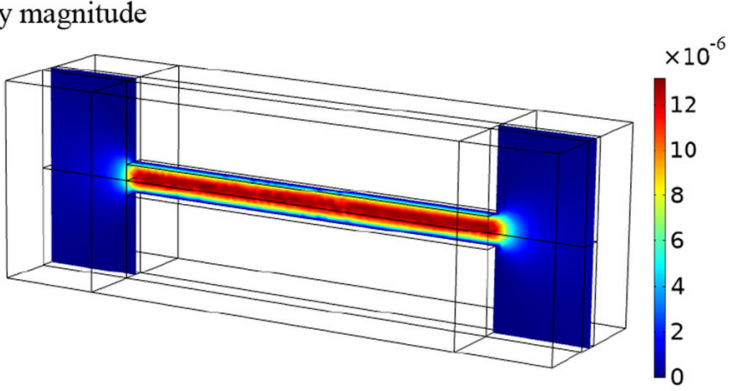

(f)

Fig. 2 a, b Scaled electric potential, $\mathbf{c}, \mathbf{d}$ scaled net charge density, and e, $\mathbf{f}$ scaled velocity field in nanopore and nanoslit are shown

\section{Results and discussions}

To systematically compare the nanopore with nanoslits, the cross section area for all cases was kept constant. The electric potential, net charge density $(n p-n n)$, and velocity created in the nanopore and nanoslit developed inside the pore/channel are shown in Fig. 2 , where the pressure difference along the pore and slit are equal. For both nanopore and nanoslit, as shown in Fig. 2, the maximum values of scaled electrical potential and net charge density occur on the walls and decrease toward the center. The velocity is zero at the walls (no-slip boundary condition) and reaches its maximum value at the center.

The fluid flow due to the pressure difference creates a perceptible and constant force in the moving part of the double layer (diffuse layer), which can cause the transfer of free ions along the flow direction. This streaming current across the pore/slit (ionic convection flux), generated by the electrokinetic flow of a dilute electrolyte solution in a nanopoe/nanoslit with charged walls, creates a magnetic field. The magnetic field inside the nanopore/nanoslit system can be affected by the transport trajectory of any biomolecules with dipoles and outside the nanoslit/nanopore system. This change can be observed as a secondary signal as a part of the sequencing technique $[46,49]$. 


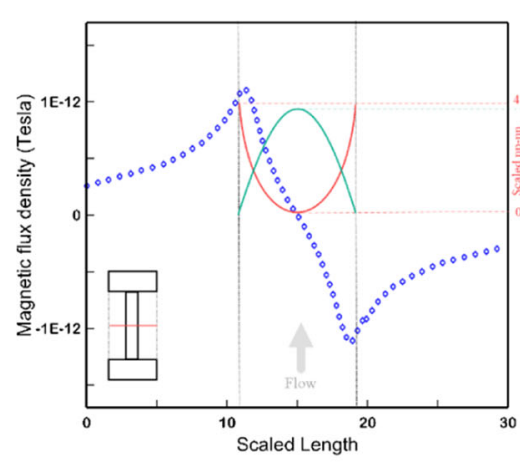

magnetic flux (z-component)

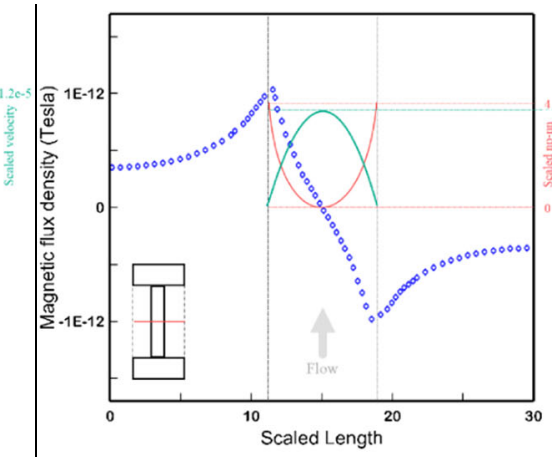

magnetic flux ( $z$-component)

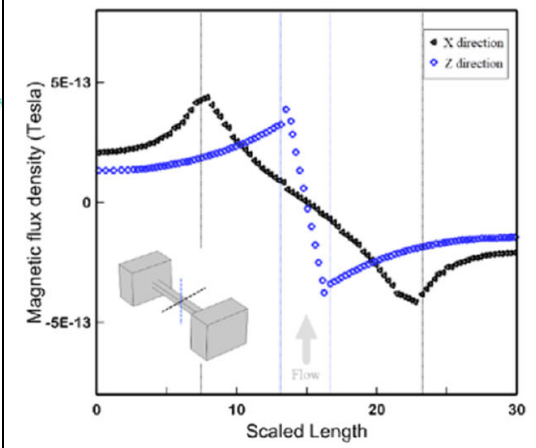

magnetic flux (z-component)

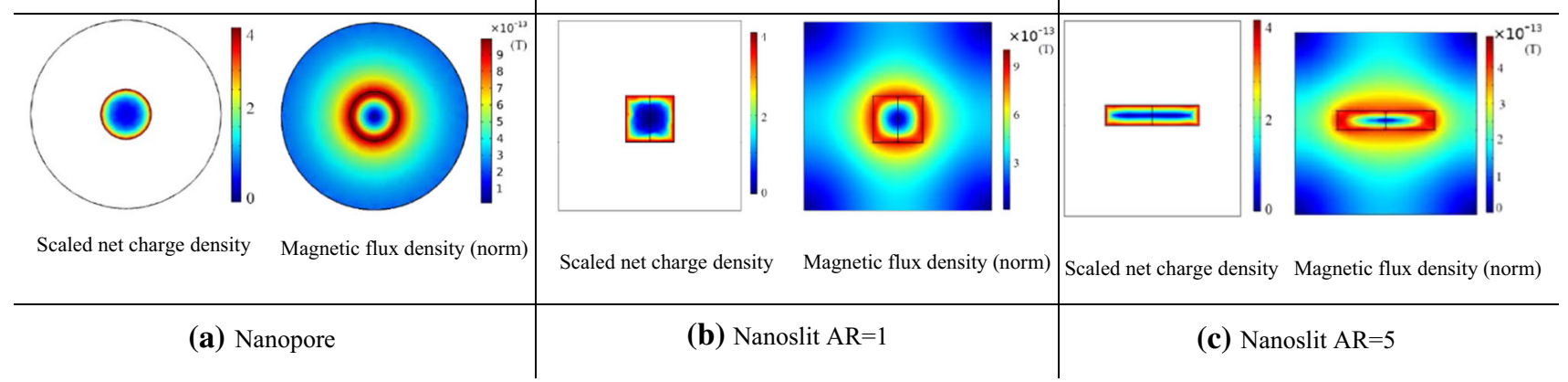

Fig. 3 Induced magnetic flux ( $z$-component in 2D plane), scaled net charge density and norm of magnetic flux density of a nanopore, $\mathbf{b}$ nanoslit ( $A R$ is 1$)$ and $\mathbf{c}$ nanoslit ( $A R$ is 5$)$ with equal cross-sectional area are compared. The pressure difference across the pore/slit is $2 \mathrm{MPa}$. For both nanopore and nanoslit, the maximum of magnetic flux density is inside the pore/slit and near the wall

The normal to the surface component of magnetic flux strength $(z$ component in 2D, see Fig. 1) inside and outside the nanoslit/nanopore system are shown in Fig. 3. Please note the $z$ component in 2D (or azimuth in 3D cylindrical coordinates) is almost equal to the norm in 3D, which indicates the other components of the magnetic flux strength are negligible compared to the $z$ component. Regarding the $z$ component in 2D (or azimuth in 3D cylindrical coordinates), as can be justified by Ampere law to a closed path, the magnetic flux at the center of nanopore/nanoslit is zero and has opposite signs around the center.

As shown in Fig. 3, the maximum value of the magnetic flux density occurs near the nanoslit/nanopore wall and slightly inside the pore/slit. To explain, in Fig. 4, the maximum flux density near a conductive wire and near the pore are compared. The magnetic flux is affected by both the net charge density and its velocity. For the pore, despite the wire, net charge density and net charge velocity are not uniform. The net charge density is higher close to the wall and decays by moving away from the wall, and its velocity which is proportional to fluid velocity is zero on the wall (due to no-slip boundary condition of fluid particles on the wall, a fluid particle in direct contact with a solid, sticks to the surface due to viscous effects, and the no slip condition, i.e., $u=0$ ) and reaches its maximum value in the center of pore. As such, considering the combined effect of net charge density and velocity, the maximum value of the magnetic flux density occurs near the wall and not exactly on the wall, see Fig. 4. In other words, for the pore/slit, there is a high density of positive ions in the stern layer, but these ions are fixed and do not contribute to ionic current and magnetic flux creation, accordingly. At the center of a current-carrying conductor of finite radius, a circle of zero radius encloses zero current, therefore there is zero magnetic field at the center.

By comparing the nanopores and nanoslits with equal cross-sectional area and same pressure difference, it was observed that the maximum magnetic flux density for nanopore is higher than that for nanoslit. And among nanoslits the maximum magnetic flux density is higher for the square cross section (i.e., $A R=1$ ). For nanoslits, by deviating from $A R=1$ (from square cross section to rectangle), the maximum magnetic flux density decreases. For example, by comparing a nanopore (diameter $8.00 \kappa^{-1}$ ), nanoslit AR $=1\left(7.09 \kappa^{-1} \times 7.09 \kappa^{-1}\right)$ and nanoslit $\mathrm{AR}=5\left(3.17 \kappa^{-1} \times 15.85 \kappa^{-1}\right)$, and pressure difference of $2 \mathrm{MPa}$ across the pore/slit, the maximum magnetic fluxes are $1.10 \mathrm{pT}, 1.08 \mathrm{pT}$, and $0.45 \mathrm{pT}$ accordingly. For the rectangular nanoslit, the maximum magnetic flux occurs near the width (shorter side of the cross section) and not near the length (see Fig. 3c).

For nanoslits, as shown above, by deviating from $A R=1$, magnetic flux density decreases. The magnetic flux and flow rate versus $A R$ are shown in Fig. 5b. By deviating from $A R=1$, given the cross section area is constant, the solid-liquid surface area increases. Increase of the solid-liquid surface area results in friction increase and flow rate decrease. At the same time, increasing the solid-liquid surface area increases the ions created on the surface. Decreasing the flow rate decreases the magnetic flux, and increasing the created ions, increases the magnetic flux. So, by deviating $A R$ from 1, two opposing factors compete against each other to change the magnetic flux. At higher pressures, the winner is the effect of flow rate decrease and by deviating from the 
Fig. 4 Schematic comparison of the magnetic flux around a conductive wire and an axisymmetric nanopore. For the pore, despite the conductive wire, the maximum magnetic flux is not on the wall (a) Magnetic field of electric currents in a conductive ivire

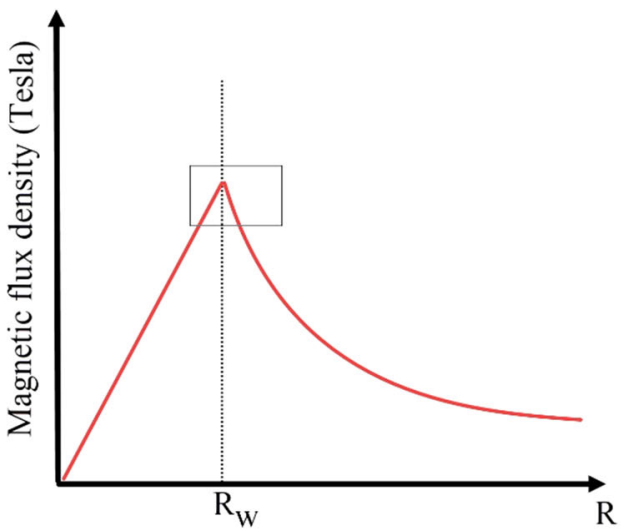

(b) Magnetic field of ionic currents in a non-conductive nanopore
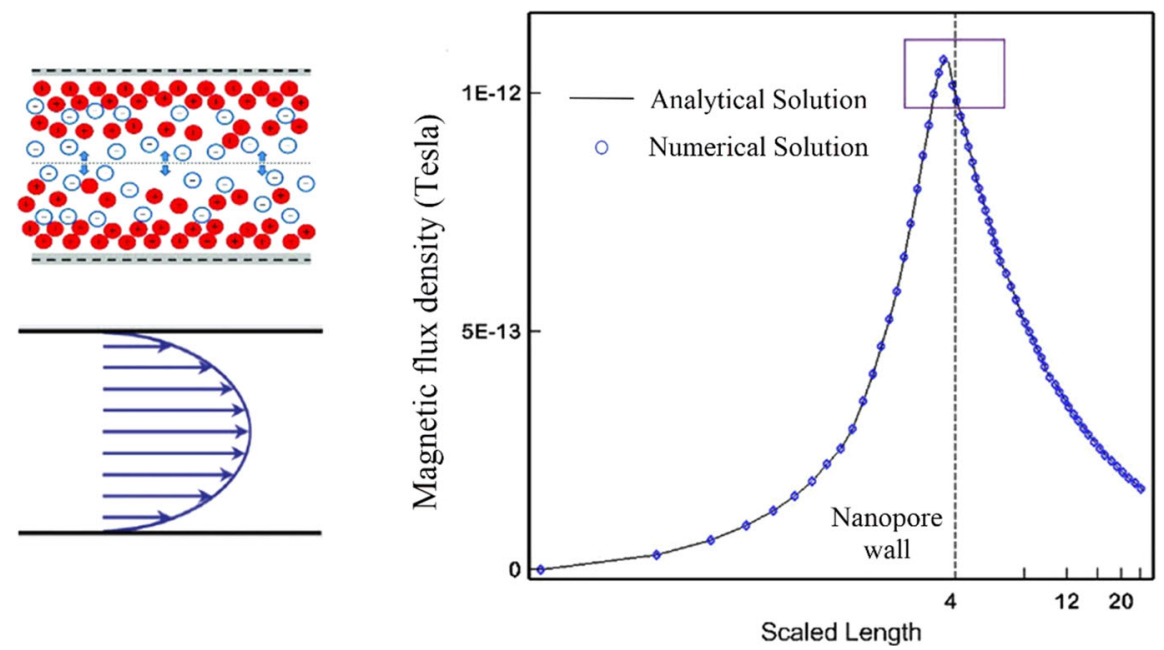

$A R=1$, magnetic flux density decreases, see Fig. 5b. Whereas at lower pressures, the two effects are comparable and by deviating the $A R$ from 1, the magnetic flux density only slightly changes, see Fig. 5b.

Increasing the magnetic flux density (i.e., the signal amplification) increases the performance range, accuracy of the detectors, and efficiency of the nanopores/nanoslits systems. One way to increase the magnetic flux is to increase the flow rate. The flow rate increase can be achieved by increasing the pressure gradient, see Fig. 5a. Therefore, it can be concluded that in practical applications, increasing the pressure gradient can be used to amplify the signals. However, increasing the inlet pressure inside the nanopore/nanoslit is limited [56].

It should be noted the external magnetic fields as small as pico-Tesla, which is of the order that is demonstrated in Figs. 3, 4 and 5, are detectable and measurable in voltage/pressure-driven electrokinetic flow nanoslits [57-60]. Therefore, we believe that the external magnetic field generated by the ionic current can be used as a secondary signal to enhancement and increase the currentbased DNA nanopore/nanoslit sequencing techniques. In other words, the existing defects that may occur during sensing the current can be reduced by sensing the magnetic field signals.

Given the pressure difference across the pore and slit is equal, the maximum magnetic flux of the nanopore becomes larger than that of the nanoslit. It is worth comparing the nanoslits and nanopores at equal flow rates. As such, we increased the pressure across the slit $(A R=1)$ by $6 \%$ to make sure the flow rate of the nanopore and nanoslit $(A R=1)$ are equal. Increasing the pressure difference increases the flow rate, electric field (Fig. 6b), and current density (Fig. 6c). Consequently, the maximum magnetic flux density of the nanoslit becomes slightly larger than that of nanopore, see Fig. 6a. Regarding Fig. 6, there is no electric field inside the nanochannel in the streaming current mode, but there are spikes with opposite signs at the inlet and outlet of the nanochannel (see Fig. 6b). The average magnitude of electric field strongly depends on the pressure gradient. The electric field induces a conduction current of ions, which in steady-state balances the convection current. The internal current density of the nanopore and nanoslit are shown in Fig. 6c, note that the maximum current density is near the wall and not exactly on the wall. 


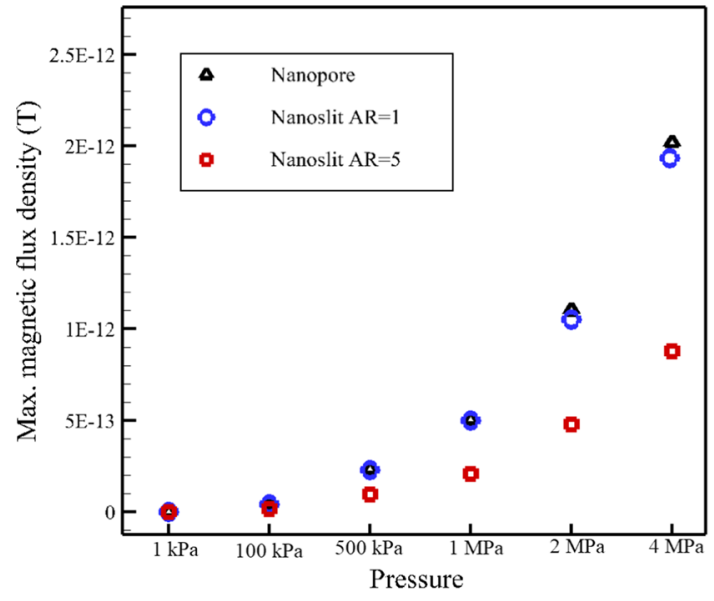

(a)

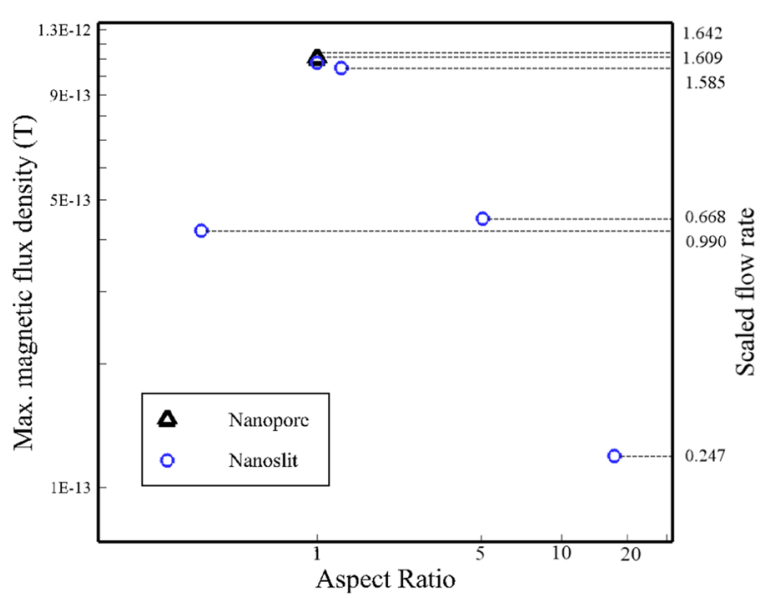

(b)

Fig. 5 Maximum magnetic flux density versus a pressure difference across the pore/slit and $\mathbf{b} A R$ where the pressure difference across the pore/slit is $2 \mathrm{MPa}$ are shown. At higher pressure differences, the maximum magnetic flux density of nanopores is higher than that of nanoslits. For all of the above cases, and the cross section area of nanopore and nanoslits are equal

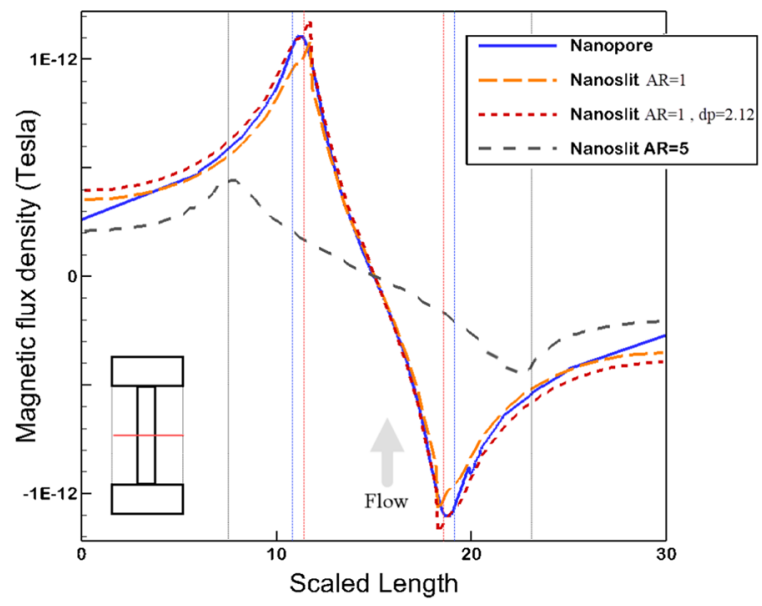

(a)

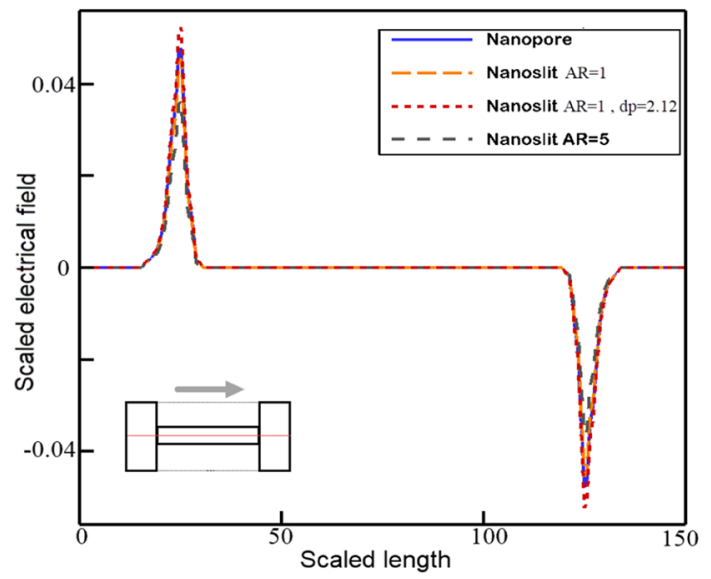

(b)

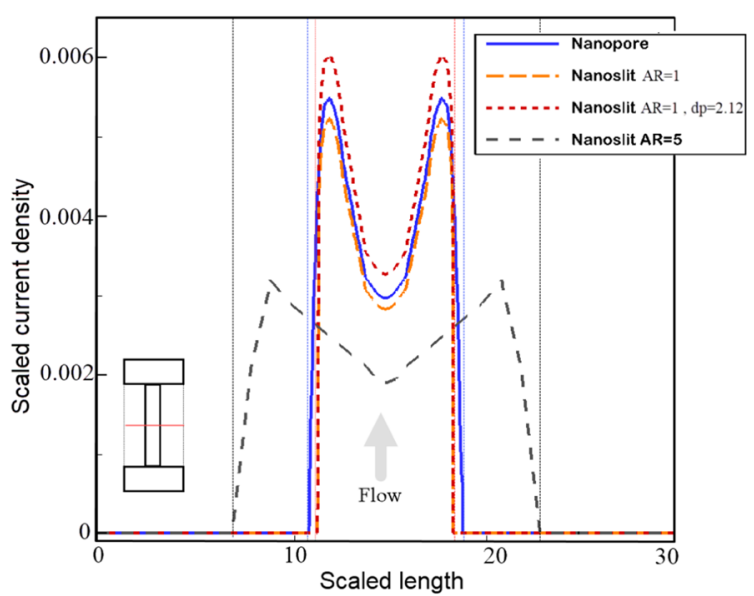

(c)

Fig. 6 a Magnetic flux density ( $\mathrm{z}$ component), b electrical field, $\mathbf{c}$ scaled current density of nanopore and nanoslit are compared. The results are taken along the line marked in the insets. For all of the cases, cross-sectional areas are kept constant. The pressure difference across the pore/slit is $2 \mathrm{MPa}$, except for $\mathrm{AR}=1, \mathrm{dp}=2.12 \mathrm{MPa}$ which the pressure across the nanoslit is $6 \%$ increased to make sure the flowrate of the slit and pore are equal 
As such, we can change a narrow section of a nanopore to nanoslit $(\mathrm{AR}=1)$ and place the magnetic flux sensor on the square section. Placing the sensor on a flat surface is easier than placing it on a curved surface, so installation becomes easier. Also, flowrate passing through the square and round sections are equal (continuity), the sensed magnetic flux density is slightly higher on the square cross section, see Fig. 6a.

\section{Conclusion}

In this study, a set of Poisson, Nernst-Planck, Ampere and Navier-Stokes governing equations (P-N-P-A-N-S) were solved to simulate induced internal and external magnetic fluxes generated by streaming currents/potentials inside 3D finite length charged nanoslit and nanopore. The nanopore and nanoslits (with various aspect ratios) with equal cross-sectional area are compared. It was observed and explained that the maximum magnetic flux density occurs near the wall and slightly inside the pore/slit. At a given pressure difference across the pore/slit, the nanopores is better than the nanoslit in sensing the magnetic field. However, at a given flowrate across the pore/slit the nanoslit performs better. For example, by applying $2 \mathrm{MPa}$ across the pore/slit with cross-sectional area of $462 \mathrm{~nm}^{2}$, and length of $303 \mathrm{~nm}$, the maximum magnetic flux on nanopore, nanoslit $A R=1$, and nanoslit $A R=5$ are $1.10 \mathrm{pT}, 1.08 \mathrm{pT}, 0.45 \mathrm{PT}$, accordingly. It should be noted the external magnetic fields as small as pico-Tesla are detectable and measurable in voltage/pressure driven electrokinetic flow slits.

Data Availability Statement This manuscript has associated data in a data repository. [Authors' comment: The datasets generated during and/or analyzed during the current study are not publicly available but are available from the corresponding author on reasonable request.]

\section{Appendix}

Validation

Both internal and external magnetic flux densities can be found analytically for a 2D infinite nanopore in streaming current mode, according to Ampere's law. The electrical state of the fluid-substrate interface is the source of streaming current that creates a spatial distribution of free charge density, $\rho_{f}$, which is then convected by the fluid, $v$. The streaming current is the product of velocity field and net charge density as follows:

$$
J(y)=v(y) \rho_{f}(y)
$$

By replacing velocity and net charge density from Navier-Stokes and Poisson equations, and in streaming current mode (no electric field) $J(y)$ becomes [54]:

$$
J(y)=-\left(\frac{\left(\frac{h}{2}\right)^{2}-y^{2}}{2 \mu}\right) \frac{\mathrm{d} p}{\mathrm{~d} y} \frac{\operatorname{Cosh}(\kappa y)}{\operatorname{Cosh}(\kappa h)} \varepsilon \xi^{2} \kappa
$$

Fig. 7 Velocity field and magnified view of parabolic velocity profile in the middle of nanoslit

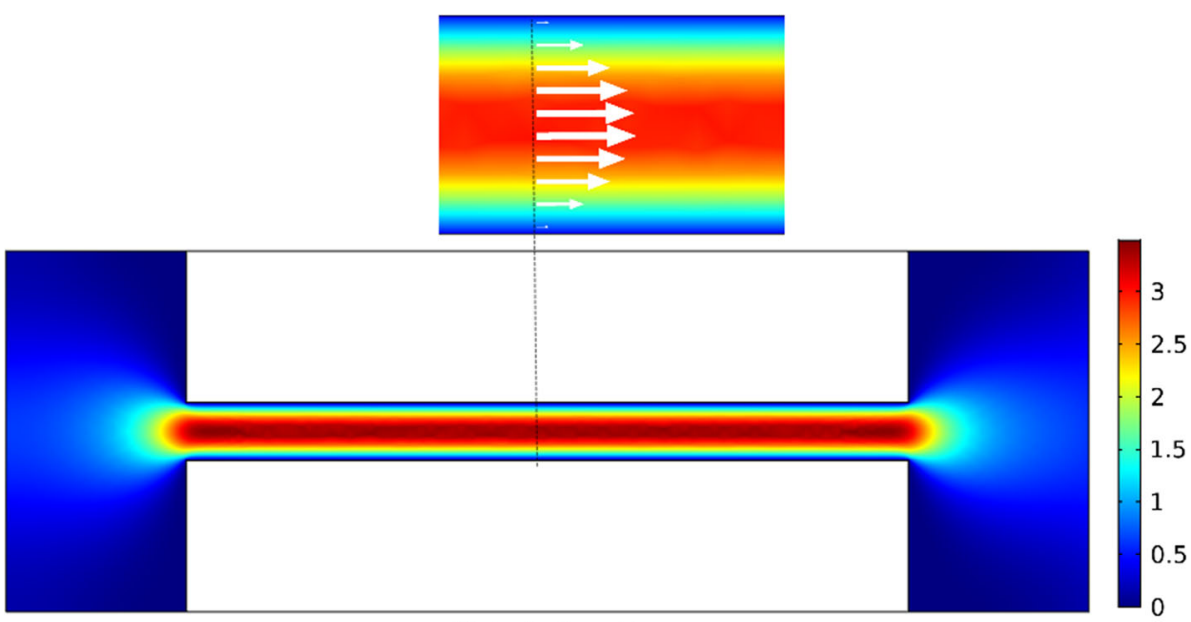

Velocity $(\mathrm{mm} / \mathrm{s})$ 
Fig. 8 Magnetic flux density inside and outside 2D nanoslit wall in streaming current mode. The inset shows the external magnetic flux density on a line parallel to centerline and outside of nanoslit. The arrow shows the direction of fluid flow

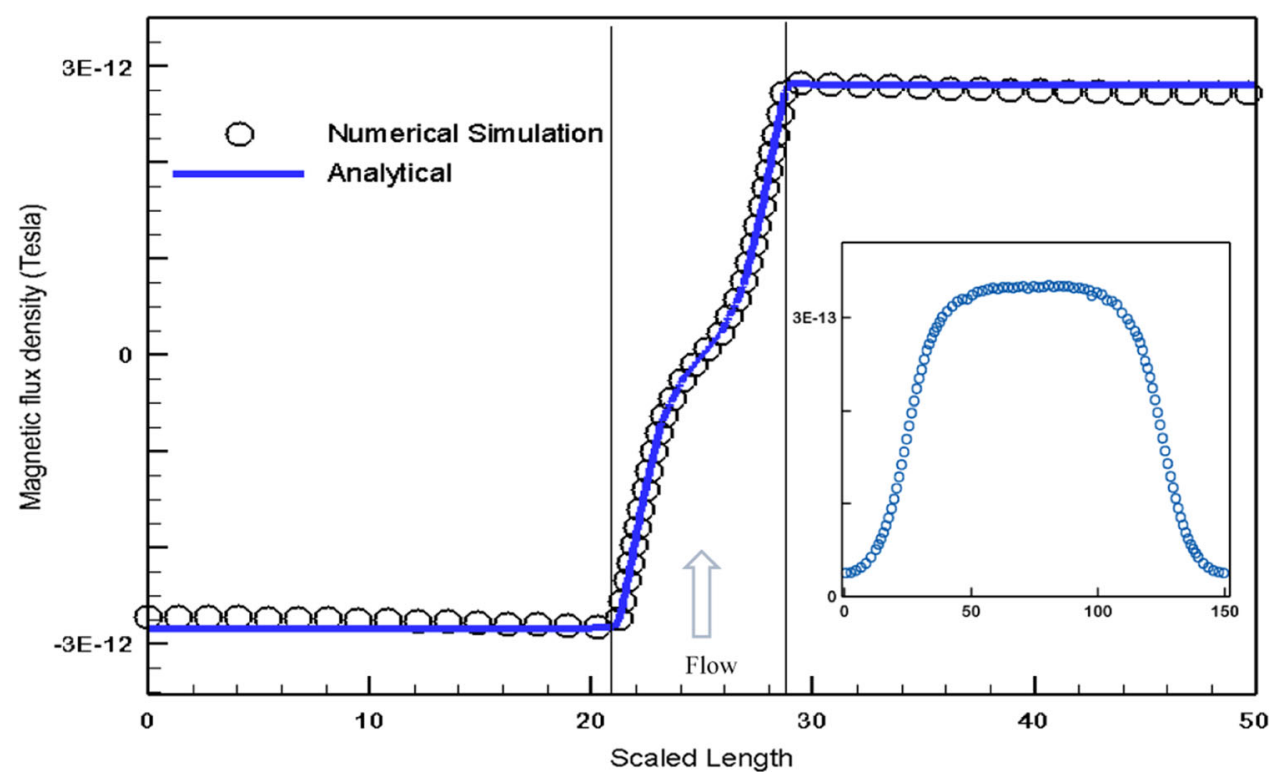

where $\varepsilon$ is permittivity, $\zeta$ is surface potential, and $\kappa$ is inverse of Debye length, [54]. To calculate the magnetic field outside an infinite nanoslit, we consider a closed path around the nanoslit cross section, according to Ampere's law and because of symmetry, the magnetic field must be fixed on both sides of the path:

$$
\oint \mathfrak{B} \cdot \mathrm{d} l=\mu_{0} I_{S C}
$$

where streaming current $\left(I_{S C}\right)$ is:

$$
I_{\mathrm{SC}}=\int J(y) \cdot \mathrm{d} A=J_{0} L h
$$

where $J_{0}$ is the current density. Therefore $\mathfrak{B}$ becomes:

$$
\mathfrak{B}= \begin{cases}\frac{\mu_{0} h}{2} J_{0}, & y>\frac{h}{2} \\ -\frac{\mu_{0} h}{2} J_{0}, & y<-\frac{h}{2}\end{cases}
$$

Inside the nanoslit, magnetic field fluctuates due to the non-uniformity of streaming current profiles, but outside the nanoslit, magnetic field does not change. To calculate the internal magnetic field inside the nanoslit, one should integrate the non-uniform streaming current density, as shown in [61]:

$$
I_{\text {inside }}=\int_{-\frac{h}{2}}^{\frac{h}{2}} J(y) \mathrm{d} y=2 \int_{0}^{\frac{h}{2}}\left(\frac{y^{2}-\left(\frac{h}{2}\right)^{2}}{2 \mu}\right) \frac{\mathrm{d} p}{\mathrm{~d} y} \frac{\operatorname{Cosh}(\kappa y)}{\operatorname{Cosh}(\kappa h)} \varepsilon \xi^{2} \kappa \mathrm{d} y
$$

And the magnetic field inside the nanoslit can be considered as follow (Figs. 7, 8):

$$
\mathfrak{B}=\frac{\mu_{0} I_{\text {inside }}}{2}
$$

\section{References}

1. B.M. Venkatesan, R. Bashir, Nanopore sensors for nucleic acid analysis. Nat. Nanotechnol. 6(10), 615-624 (2011)

2. S.M. Avdoshenko, D. Nozaki, C. Gomes Da Rocha, J.W. González, M.H. Lee, R. Gutierrez et al., Dynamic and electronic transport properties of DNA translocation through graphene nanopores. Nano Lett. 13(5), 1969-1976 (2013)

3. A.J. Storm, C. Storm, J. Chen, H. Zandbergen, J.F. Joanny, C. Dekker, Fast DNA translocation through a solid-state nanopore. Nano Lett. 5(7), 1193-1197 (2005)

4. M. Wanunu, J. Sutin, B. McNally, A. Chow, A. Meller, DNA translocation governed by interactions with solid-state nanopores. Biophys. J. 95(10), 4716-4725 (2008)

5. J.J. Kasianowicz, E. Brandin, D. Branton, D.W. Deamer, Characterization of individual polynucleotide molecules using a membrane channel, in Proceedings of the National Academy of Sciences of the United States of America. National Academy of Sciences (1996), pp. 13770-13773

6. K. Chen, M. Juhasz, F. Gularek, E. Weinhold, Y. Tian, U.F. Keyser et al., Ionic current-based mapping of short sequence motifs in single DNA molecules using solid-state nanopores. Nano Lett. 17(9), 5199-5205 (2017) 
7. D. Branton, D.W. Deamer, A. Marziali, H. Bayley, S.A. Benner, T. Butler et al., The potential and challenges of nanopore sequencing. Nat. Biotechnol. 26(10), 1146-1153 (2008)

8. C.C.Y. Yip, W.M. Chan, J.D. Ip, C.W.M. Seng, K.H. Leung, R.W.S. Poon et al., Nanopore sequencing reveals novel targets for detection and surveillance of human and avian influenza a viruses. J. Clin. Microbiol. 58(5), 1056 (2020)

9. S.C.B. Stubbs, B.A. Blacklaws, B. Yohan, F.A. Yudhaputri, R.F. Hayati, B. Schwem, et al., Assessment of a multiplex PCR and Nanopore-based method for dengue virus sequencing in Indonesia

10. O. Behrmann, M. Spiegel, Comment COVID-19: from rapid genome sequencing to fast decisions (2020)

11. X. Zhao, D. Wang, Y. Zhao, Y. Deng, D. Zhou, Z. Zhang et al., Hybridized plasmons in 2D nanoslits: from graphene to anisotropic 2D materials. ACS Photonics 7(May), 384-387 (2018)

12. J. Comer, A. Aksimentiev, DNA sequence-dependent ionic currents in ultra-small solid-state nanopores. Nanoscale 8(18), 9600-9613 (2016)

13. J.M. Park, Y.E. Pak, H. Chun, J.H. Lee, 3-D simulation of nanopore structure for DNA sequencing. J. Nanosci. Nanotechnol. 12(7), 5160-5163 (2012)

14. M.R. Hasan, M. Arif, I. Mahmood, R.R. Khanzada, N. Mansur, A. Adnan, et al., One central press molecular dynamics study of protein deformation through solid-state nanopore

15. P.B. Peters, R. Van Roij, M.Z. Bazant, P.M. Biesheuvel, Analysis of electrolyte transport through charged nanopores. Phys. Rev. E 93(5), 053108 (2016)

16. R.B. Schoch, H. van Lintel, P. Renaud, Effect of the surface charge on ion transport through nanoslits. Phys. Fluids 17(10), 100604 (2005)

17. A.H. Christensen, H.A. Stone, K.H. Jensen, Diffusion and flow across shape-perturbed plasmodesmata nanopores in plants. Eur. Phys. J. Plus 136(8), $1-15(2021)$

18. M. Waugh, K. Briggs, D. Gunn, M. Gibeault, S. King, Q. Ingram et al., Solid-state nanopore fabrication by automated controlled breakdown. Nat. Protoc. 15(1), 122-143 (2020)

19. K. Lee, K.B. Park, H.J. Kim, J.S. Yu, H. Chae, H.M. Kim et al., Recent progress in solid-state nanopores. Adv. Mater. 30(42), 1-28 (2018)

20. R. Dela Torre, J. Larkin, A. Singer, A. Meller, Fabrication and characterization of solid-state nanopore arrays for high-throughput DNA sequencing. Nanotechnology 23(38), 385308 (2012)

21. J. Lee, S. Kim, H. Jeong, G.Y. Jung, R. Chang, Y.L. Chen et al., Nanoslit confined DNA at low ionic strengths. ACS Macro Lett. 3(9), 926-930 (2014)

22. E.L. Bonome, F. Cecconi, M. Chinappi, Electroosmotic flow through an $\alpha$-hemolysin nanopore. Microfluid Nanofluidics 21(5), 1-9 (2017)

23. W. Yang, B. Radha, A. Choudhary, Y. You, G. Mettela, A.K. Geim et al., Translocation of DNA through ultrathin nanoslits. Adv. Mater. 33(11), 2007682 (2021)

24. C. Huang, X. Zhu, Z. Li, X. Ma, N. Li, J. Luo et al., Molecular insights into geometric and electrophoretic effects on DNA translocation speed through graphene nanoslit sensor. SSRN Electron. J. 25, 25 (2021)

25. H.Y. Hsieh, R. Chang, Y.Y. Huang, P.H. Juan, H. Tahara, K.Y. Lee et al., Continuous polymerase chain reaction microfluidics integrated with a gold-capped nanoslit sensing chip for Epstein-Barr virus detection. Biosens. Bioelectron. 195, 113672 (2022)

26. M. Wanunu, Nanopores: a journey towards DNA sequencing. Phys. Life Rev. 9, 125-158 (2012)

27. D. Branton, D.W. Deamer, A. Marziali, H. Bayley, S.A. Benner, T. Butler, et al., The potential and challenges of nanopore sequencing, in Nanoscience and Technology: A Collection of Reviews from Nature Journals (World Scientific Publishing Co., 2009), pp. 261-268

28. W. Shi, A.K. Friedman, L.A. Baker, Nanopore sensing. Anal. Chem. 89, 157-188 (2017)

29. A.J. Storm, J.H. Chen, H.W. Zandbergen, C. Dekker, Translocation of double-strand DNA through a silicon oxide nanopore. Phys. Rev. E Stat. Nonlinear Soft Matter Phys. 71(5), 1-10 (2005)

30. Z. Chen, Y. Jiang, D.R. Dunphy, D.P. Adams, C. Hodges, N. Liu et al., DNA translocation through an array of kinked nanopores. Nat. Mater. 9(8), 667-675 (2010)

31. A. Pomposo, J. Rubio-cervilla, E. Gonzalez, A.J. Moreno, A. Arbe, J. Colmenero, Ultrafiltration of single-chain polymer nanoparticles through nanopores and nanoslits. Polymer 148, 61-67 (2018)

32. M. Kwiat, D. Stein, F. Patolsky, Nanotechnology meets electrophysiology. Curr. Opin. Biotechnol. 24, 654-663 (2013)

33. M.V. Accardi, M.K. Pugsley, R. Forster, E. Troncy, H. Huang, S. Authier, The emerging role of in vitro electrophysiological methods in CNS safety pharmacology. J. Pharmacol. Toxicol. Methods 81, 47-59 (2016)

34. N. Modi, M. Winterhalter, U. Kleinekathöfer, Computational modeling of ion transport through nanopores. Nanoscale R. Soc. Chem. 4, 6166-6180 (2012)

35. L.J. Steinbock, A. Radenovic, The emergence of nanopores in next-generation sequencing. Nanotechnology 26(7), 074003 (2015)

36. M.E. Gracheva, A. Xiong, A. Aksimentiev, K. Schulten, G. Timp, J.P. Leburton, Simulation of the electric response of DNA translocation through a semiconductor nanopore-capacitor. Nanotechnology 17(3), 622-633 (2006)

37. D. Deamer, M. Akeson, D. Branton, Three decades of nanopore sequencing. Nat. Biotechnol. 34(5), 518-524 (2016)

38. Y. He, M. Tsutsui, C. Fan, M. Taniguchi, T. Kawai, Controlling DNA translocation through gate modulation of nanopore wall surface charges. ACS Nano 5(7), 5509-5518 (2011)

39. Y. Tsuyama, K. Mawatari, Characterization of optical diffraction by single nanochannel for aL-fL sample detection in nanofluidics. Microfluid. Nanofluid. 24(4), 1-8 (2020)

40. B. Lu, D.P. Hoogerheide, Q. Zhao, H. Zhang, Z. Tang, D. Yu et al., Pressure-controlled motion of single polymers through solid-state nanopores. Nano Lett. 13(7), 3048-3052 (2013)

41. H. Zhang, Q. Zhao, Z. Tang, S. Liu, Q. Li, Z. Fan et al., Slowing down DNA translocation through solid-state nanopores by pressure. Small 9(24), $4112-4117(2013)$

42. K.J. Freedman, L.M. Otto, A.P. Ivanov, A. Barik, S.H. Oh, J.B. Edel, Nanopore sensing at ultra-low concentrations using single-molecule dielectrophoretic trapping. Nat. Commun. 7, 1-9 (2016)

43. F.J. Rang, W.P. Kloosterman, J. de Ridder, From squiggle to basepair: computational approaches for improving nanopore sequencing read accuracy. Genome Biol. 19, 1-11 (2018)

44. D.P. Hoogerheide, B. Lu, J.A. Golovchenko, Pressure-voltage trap for DNA near a solid-state nanopore. ACS Nano 8(7), 7384-7391 (2014)

45. H. Zhang, Q. Chen, Y. Wu, Y. Wang, X. Bei, L. Xiao, The temporal resolution and single-molecule manipulation of a solid-state nanopore by pressure and voltage. Nanotechnology 29(49), 495501 (2018)

46. A. Mansouri, P. Taheri, L.W. Kostiuk, Streaming current magnetic fields in a charged nanopore. Sci. Rep. 6(November), 1-10 (2016)

47. A. Mansouri, A. Tarokh, P. Taheri, Ionic current magnetic fields in a two dimensional nanoslit. Chem. Phys. Lett. 710, 150-156 (2018)

48. H. Daiguji, P. Yang, A. Majumdar, Ion transport in nanofluidic channels. Nano Lett. 4(1), 137-142 (2004)

49. A. Mansouri, S. Bhattacharjee, L.W. Kostiuk, Transient electrokinetic transport in a finite length microchannel: currents, capacitance, and an electrical analogy. J. Phys. Chem. B 111(44), 12834-12843 (2007). https://doi.org/10.1021/jp074386c

50. A. Mansouri, C. Scheuerman, S. Bhattacharjee, D.Y. Kwok, L.W. Kostiuk, Transient streaming potential in a finite length microchannel. J. Colloid Interface Sci. 292(2), 567-580 (2005) 
51. A. Mansouri, L.W. Kostiuk, S. Bhattacharjee, Streaming current measurements in a glass MicroChannel array. J. Phys. Chem. C 112(42), 16192-16195 (2008)

52. R. Probstein, Physicochemical hydrodynamics: an introduction (2005)

53. B. Ali Rachedi, A. Babouri, F Berrouk, A study of electromagnetic field generated by high voltage lines using COMSOL MULTIPHYSICS, in 2014 Int Conf Electr Sci Technol Maghreb, Cist 2014 (2014)

54. D. Burgreen, F.R. Nakache, Electrokinetic flow in ultrafine capillary slits. J. Phys. Chem. 68(5), 1084-1091 (1964)

55. S. Chaudhuri, S. Sahoo, Effect of the aspect ratio on the flow characteristics of magnetohydrodynamic (MHD) third grade fluid flow through a rectangular channel. Sadhana Acad. Proc. Eng. Sci. 43(7), 1-10 (2018)

56. Z. Roelen, J.A. Bustamante, A. Carlsen, A. Baker-Murray, V. Tabard-Cossa, Instrumentation for low noise nanopore-based ionic current recording under laser illumination. Rev. Sci. Instrum. 89(1), 897 (2018)

57. G. Fabbri, C. Boccaletti, G. Fabbri, C. Boccaletti, A.J. Marques Cardoso, F. Castrica, A bioelectrical sensor for the detection of small biological currents (2010)

58. B. Reid, R. Nuccitelli, M. Zhao, Non-invasive measurement of bioelectric currents with a vibrating probe. Nat. Protoc. 2(3), 661-669 (2007)

59. N. Haned, M. Missous, Nano-tesla magnetic field magnetometry using an InGaAs-AlGaAs-GaAs 2DEG Hall sensor. Sensors Actuators A Phys. 102(3), 216-222 (2003)

60. J. Zhai, Z. Xing, S. Dong, J. Li, D. Viehland, Detection of pico-Tesla magnetic fields using magneto-electric sensors at room temperature. Appl. Phys. Lett. 88(6), 062510 (2006)

61. W. Hayt, J. Buck, Engineering electromagnetics (1981) 